\title{
Effects of the Framework and Mesoporosity on the Catalytic Activity of Hierarchical Zeolite Catalysts in Benzyl Alcohol Conversion
}

\author{
Chao $\mathrm{Li}^{[\mathrm{a}, \mathrm{b}]}$ Hong Je Cho, ${ }^{[\mathrm{b}]}$ Zhuopeng Wang, ${ }^{[\mathrm{b}, \mathrm{c}]}$ Jinsheng Gou, ${ }^{[\mathrm{b}, \mathrm{d}]}$ Yanqun Ren, ${ }^{[\mathrm{a}]}$ \\ Hongxia $\mathrm{Xi}^{*}{ }^{[\mathrm{a}]}$ and Wei Fan ${ }^{*[\mathrm{~b}]}$
}

\begin{abstract}
The catalytic performance of three-dimensionally ordered mesoporous imprinted (3DOm-i) zeolite catalysts with different frameworks (3DOm-i MFI, 3DOm-i BEA, and 3DOm-i LTA) were investigated by the liquid-phase catalytic conversion of benzyl alcohol in mesitylene and compared to that of other microporous and mesoporous catalysts with a high external surface area, which included MCM-22, $300 \mathrm{~nm} \mathrm{MFI,} \mathrm{ITQ-2,} \mathrm{and} \mathrm{Al-MCM-}$ 41. The mesoporosity in MFI and BEA zeolites can effectively enhance the catalytic performance of the zeolite catalysts for
\end{abstract}

benzyl alcohol self-etherification catalyzed by both the acid sites on the external and internal surface and the alkylation of mesitylene with benzyl alcohol catalyzed exclusively by the acid sites on the external surface. For 3DOm-i LTA, MCM-22, and Al-MCM-41, only the acid sites on the external surface can be utilized in the catalytic reactions. A distinct difference in the product selectivity was also observed for the microporous and mesoporous catalysts.

\section{Introduction}

Zeolites are crystalline inorganic porous materials formed by $\mathrm{TO}_{4}$ tetrahedra ( $\mathrm{T}=\mathrm{Si}, \mathrm{Al}, \mathrm{P}$, etc.) with ordered microporous structures. ${ }^{[1]}$ As a result of their tunable acidities and framework structures, zeolites have been used widely in catalysis and molecular adsorption/separation as well as several other emerging applications. ${ }^{[1 a, 2]}$ However, the pore size of a zeolite is usually smaller than $2 \mathrm{~nm}$, which often causes severe diffusion limitations for catalytic reactions that involve bulky molecules. The introduction of intracrystalline mesopores within zeolites to form hierarchical porous structures is a promising way to enhance the mass transport properties and catalytic performance of zeolite catalysts. ${ }^{[1,3]}$

[a] Dr. C. Li, Dr. Y. Ren, Prof. H. Xi

School of Chemistry and Chemical Engineering

South China University of Technology

No. 381 Wushan Road, Guangzhou 510640 (P.R. China)

E-mail: cehxxi@scut.edu.cn

[b] Dr. C. Li, Dr. H. J. Cho, Dr. Z. Wang, Prof. J. Gou, Prof. W. Fan

Department of Chemical Engineering

University of Massachusetts, Amherst

159 Goessmann Lab, 686 North Pleasant Street, Amherst MA, 01003-9303 (USA)

E-mail:wfan@ecs.umass.edu

[c] Dr. Z. Wang

Department of Chemistry

College of Sciences

Northeastern University

No.3-11, Culture Road, Shenyang, Liaoning 110819 (P.R. China)

[d] Prof. J. Gou

College of Material Science and Technology

Beijing Forestry University

No.35 Tsinghua East Road, Beijing 100083 (P.R. China)

$\square$ Supporting information for this article can be found under http:// dx.doi.org/10.1002/cctc.201600322.

Various synthesis approaches have been developed to fabricate mesoporous zeolites, such as the exfoliation or pillaring of layered zeolites, ${ }^{[4]}$ desilication or demetalation of zeolites by selective dissolution, ${ }^{[5]}$ and soft- and hard-templating methods. ${ }^{[6]}$ Delaminated zeolites, such as ITQ-2, which result from the delamination of a layered zeolite precursor, MCM-22(P), exhibit thin zeolite sheets $(\sim 2.5 \mathrm{~nm}$ thick) with a high external surface area $\left(>700 \mathrm{~m}^{2} \mathrm{~g}^{-1}\right) .^{[4 \mathrm{~b}]}$ However, this method is only applicable for materials composed of layered zeolite precursors. With the use of the soft-templating synthesis strategy, mesopores can be introduced successfully into zeolite crystals through the coherent assembly of zeolite precursors and specially designed structure-directing agents (SDAs). Ryoo and co-workers designed a series of diquaternary ammonium-type surfactants with long hydrophobic hydrocarbon chains to synthesize zeolites with ultrathin nanosheets. ${ }^{[4 a, 6 a, 7]}$ Tsapatsis and co-workers reported the one-step synthesis of a self-pillared pentasil (SPP) zeolite composed of orthogonally connected single-unit cell lamellae. ${ }^{[8]}$ Three-dimensionally ordered mesoporous imprinted (3DOm-i) zeolites synthesized using 3DOm carbon as a mesoscale hard template was synthesized using a hard-templating synthesis strategy. ${ }^{[6 \mathrm{~b}, 9]}$

Hierarchical zeolites with intracrystalline mesopores can provide easy access to the active sites located within micropores and fast mass transport that can improve the activity, selectivity, and lifetime of zeolite catalysts for a wide range of catalytic reactions. ${ }^{[10]}$ Although a series of breakthroughs have demonstrated the enhanced catalytic properties of hierarchical zeolites, structure-property relationships for hierarchical zeolites have not been fully established yet. ${ }^{[1 b, 11]}$ There are several critical issues that limit the rational development of these materials for catalytic reactions. These include (1) the lack of facile 
methods for the synthesis of hierarchical zeolites with accessible and controllable mesopores, (2) the elusive contribution from the active sites on the external surface of hierarchical zeolites, and (3) the unclear mass transport behavior of hierarchical zeolites and their influences on catalyst performances.

Among these hierarchical zeolites synthesized by different approaches, 3DOm-i zeolites are ideal model materials to develop a fundamental understanding of the differences in the catalytic activities of active sites in hierarchical zeolites because of their highly ordered and tunable mesoporous structures, framework compositions, and wide range of available framework structures. In this study, we evaluated the accessibility and catalytic activities of a series of 3DOm-i zeolites with different topologies (e.g., MFI, LTA, and BEA) and compared them to other conventional zeolites with a high external surface area such as MWW zeolite (MCM-22 and ITQ-2) and MFI nanocrystals. Amorphous mesoporous Al-MCM-41 was also used in this study. The catalytic properties of the Brønsted acid sites on the external and internal surface of these catalysts were investigated by the liquid-phase catalytic conversion of benzyl alcohol in mesitylene. The hierarchical zeolite catalysts exhibited enhanced catalytic performance and significant differences in product selectivity, which is controlled by both mesoporosity and microporosity.

\section{Results and Discussion}

\section{Structural properties of the catalysts}

The zeolite catalysts studied in this work include materials with small, medium, and large micropores. The topologies and pore systems of the zeolite catalysts used in this study are summarized in Table 1. LTA, a typical small-pore zeolite, is built by connecting sodalite cages with double four-membered rings (D4R), which creates a cage accessible through eight-membered rings ( $8 \mathrm{MR})$. MFI zeolite possesses an anisotropic framework with two intersecting 10-membered-ring (10 MR) channels. The straight channel is parallel to the $b$ axis, and the zigzag channel with an estimated pore opening of $0.51 \mathrm{~nm} \times$ $0.55 \mathrm{~nm}$ is parallel to the $a$ axis. The two channels along the $b$ and $a$ directions are interconnected to form three-dimensionally interconnected microporous structures. BEA is a large-

\begin{tabular}{|c|c|c|c|}
\hline \multirow[t]{2}{*}{ Catalyst } & \multirow[t]{2}{*}{ Framework } & \multicolumn{2}{|c|}{ Pore structure } \\
\hline & & Pore shape & Pore size $[\AA]$ \\
\hline 3DOm-i MFI & MFI & $10 \mathrm{MR}$ & $5.1 \times 5.5,5.3 \times 5.6^{[a]}$ \\
\hline 3DOm-i LTA & LTA & $8 \mathrm{MR}$ & $4.1 \times 4.1^{[\mathrm{a}]}$ \\
\hline 3DOm-i BEA & BEA & $12 \mathrm{MR}$ & $7.7 \times 6.6,5.6 \times 5.6^{[b]}$ \\
\hline MCM-22 & MWW & $10 \mathrm{MR}$ & $4.0 \times 5.5,4.1 \times 5.1^{[a]}$ \\
\hline $300 \mathrm{~nm}$ MFI & $\mathrm{MFI}$ & $10 \mathrm{MR}$ & $5.1 \times 5.5,5.3 \times 5.6^{[a]}$ \\
\hline ITQ-2 & N.A. & $10 \mathrm{MR}$ & $4.1 \times 5.1^{[\mathrm{a}]}$ \\
\hline Al-MCM-41 & amorphous & $\begin{array}{l}\text { hexagonal } \\
\text { mesopores }\end{array}$ & $\approx 40^{[c]}$ \\
\hline \multicolumn{4}{|c|}{$\begin{array}{l}\text { [a] Ref. [12a]. [b] Ref. [12b]. [c] Obtained from } \mathrm{N}_{2} \text { physisorption isotherm } \\
\text { based on the NLDFT method. }\end{array}$} \\
\hline
\end{tabular}

pore zeolite that exhibits 12 -membered rings ( $12 \mathrm{MR}$ ), and is composed of an intersection of $0.77 \mathrm{~nm} \times 0.66 \mathrm{~nm}$ and a tortuous channel of $0.56 \mathrm{~nm} \times 0.56 \mathrm{~nm}$. MCM-22 has an MWW framework structure that possesses a unique pore structure of 12 MR side "cups" on the crystal exterior, two independent $10 \mathrm{MR}$ channel systems along the $a$ and $b$ axes, and ultra-small six-membered ring (6 MR) transport-limiting apertures along the $c$ axis. ITQ-2 is a 2D zeolite made from the delamination of MCM-22 precursors with $12 \mathrm{MR}$ side cups on the external surface of the $2 \mathrm{D}$ sheets $(\sim 2.5 \mathrm{~nm}$ thick), these cups have an aperture of approximately $0.7 \mathrm{~nm}$ and meet at the center of the sheets and connect with a double $6 \mathrm{MR}$ window. As a result, ITQ-2 has a high external surface area $\left(>700 \mathrm{~m}^{2} \mathrm{~g}^{-1}\right)$ and affords reactants nearly unrestricted access to the acid sites on the surface. ${ }^{[4 b, 13]}$ Aluminosilicate MCM-41(AI-MCM-41) is a mesoporous material from the M41S family with a hexagonal mesopore structure.

The wide-angle powder XRD patterns of the zeolite catalysts used in this study are shown in Figure $1 \mathrm{a}$ and Figure S1. These patterns exhibit typical diffraction peaks for the MFI, LTA, BEA, MCM-22, and ITQ-2 structures, consistent with previous reports. ${ }^{[4 b, 6 b, 9]}$ Broad diffraction peaks were observed for 3DOmi BEA because of the highly intergrown zeolite BEA structure composed of different polymorphs. The small-angle XRD pat-
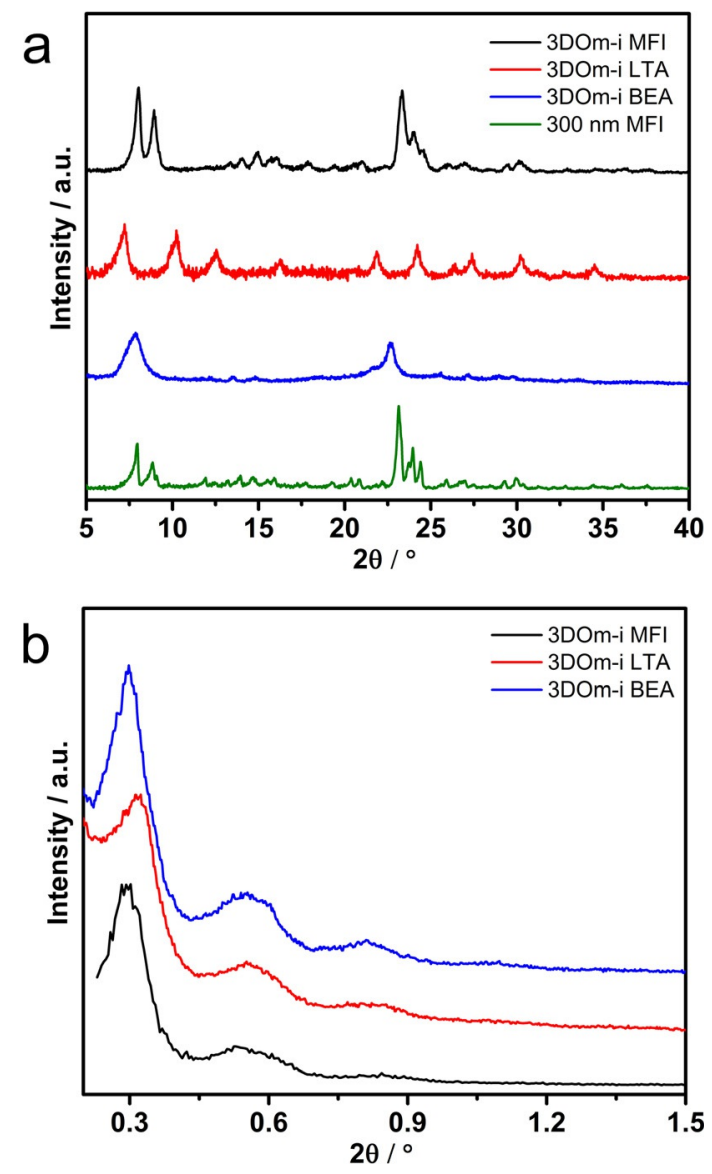

Figure 1. Crystalline structure of the zeolite catalysts. a) Wide-angle XRD patterns of 3DOm-i MFI, 3DOm-i LTA, 3DOm-i BEA, and $300 \mathrm{~nm} \mathrm{MFI}$ and b) small-angle XRD patterns of 3DOm-i MFI, 3DOm-i LTA, and 3DOm-i BEA. 
terns of the 3DOm-i zeolites are shown in Figure $1 \mathrm{~b}$ and exhibit well-resolved characteristic reflections for typical mesostructural ordering. This implies that the 3DOm-i catalysts retain a high degree of three-dimensionally ordered mesoporous ordering imprinted from the 3DOm carbon. According to a facecentered cubic (FCC) structure, the low-angle diffraction peaks were indexed. The center-to-center distance between spherical elements calculated from the peak of (111) is $(40 \pm 5) \mathrm{nm}$, which is in agreement with the pore size of the 3DOm carbon template and the TEM images shown in Figure 2. In addition,
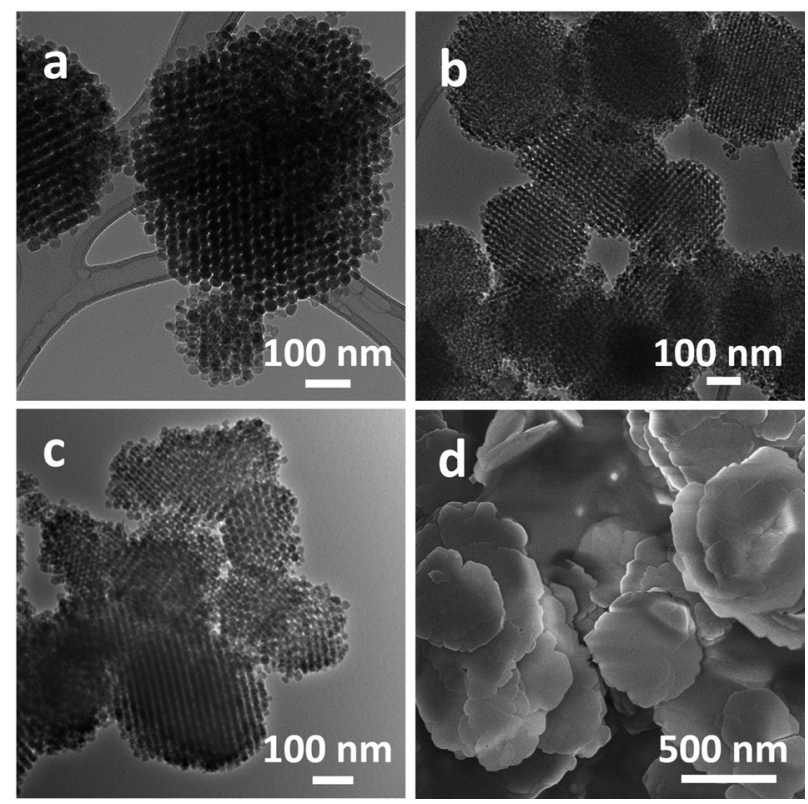

Figure 2. Morphologies of the zeolite catalysts. TEM images of a) 3DOmi MFI, b) 3DOm-i LTA, and c) 3DOm-i BEA and d) SEM image of MCM-22.

the low-angle diffraction peaks show a minor difference in the three 3DOm-i zeolites, which is most likely because of the small variation in the structures of the $3 \mathrm{DOm}$ carbon templates synthesized from different batches. MFI nanocrystal $(300 \mathrm{~nm}$ $\mathrm{MFI}$ ) has a similar XRD pattern to 3DOm-i MFI (Figure $1 \mathrm{a}$ ). ITQ2 was made from the delamination of MCM-22 precursors. The absence of the diffraction peaks of the $(001)$ and (002) planes in the XRD pattern of ITQ-2 (Figure S1 a) indicates clearly that the MCM-22 precursors were delaminated. ${ }^{[4 c, 14]}$

Compared to the XRD pattern of MCM-22, that of ITQ-2 exhibits much broader peaks at a high angle, consistent with the fact that MCM-22 precursors was delaminated into 2D nanosheet structures. The XRD pattern of Al-MCM-41 suggests that it possesses a hexagonal mesoporous structure (Figure $\mathrm{S} 1 \mathrm{~b}$ ).

The $\mathrm{N}_{2}$ physisorption isotherms measured at $77 \mathrm{~K}$ (Figure 3) show a sharp increase in the relative pressure range $\left(P / P_{0}\right)$ from 0.6 to 0.8 in the adsorption branch and a clear hysteresis because of capillary condensation, which confirms the presence of mesopores in the 3DOm-i catalysts. Mesopore size distributions calculated from the adsorption branch of the isotherms using nonlocal density functional theory (NLDFT) indicate that the pore size is centered at $6-9 \mathrm{~nm}$ with a narrow

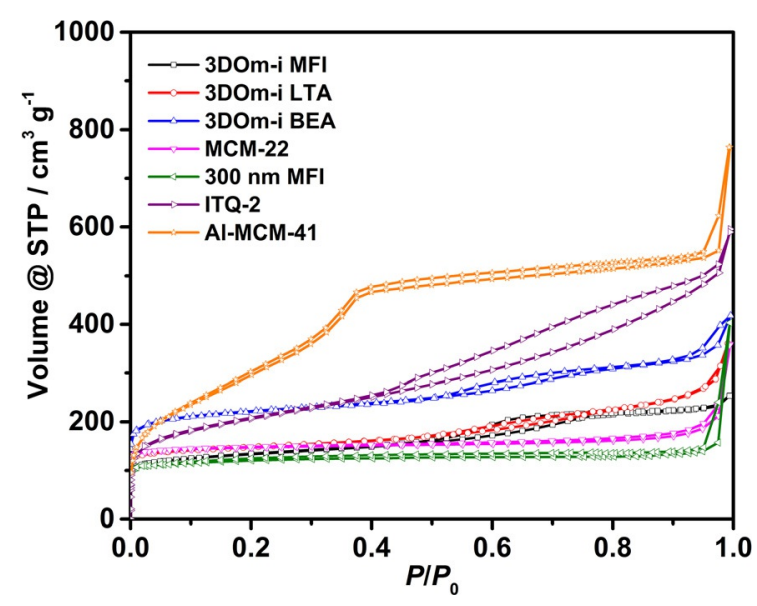

Figure 3. $\mathrm{N}_{2}$ physisorption isotherms of the zeolite catalysts.

pore size distribution (Figure S3). The textural characteristics of the catalysts, which include the BET specific surface area, external surface area, micropore volume, and total pore volume, are summarized in Table 2 . The BET specific surface areas $\left(S_{\mathrm{BET}}\right)$ of

\begin{tabular}{|c|c|c|c|c|}
\hline Catalyst & $\begin{array}{l}S_{\mathrm{BET}} \\
{\left[\mathrm{m}^{2} \mathrm{~g}^{-1}\right]}\end{array}$ & $\begin{array}{l}S_{\text {ext }}{ }^{[\mathrm{a}]} \\
{\left[\mathrm{m}^{2} \mathrm{~g}^{-1}\right]}\end{array}$ & $\begin{array}{l}V_{\text {mic }}{ }^{\text {[a] }} \\
{\left[\mathrm{cm}^{3} \mathrm{~g}^{-1}\right]}\end{array}$ & $\begin{array}{l}V_{\text {total }}^{[\mathrm{b}]} \\
{\left[\mathrm{cm}^{3} \mathrm{~g}^{-1}\right]}\end{array}$ \\
\hline 3DOm-i MFI & 477 & 230 & 0.14 & 0.43 \\
\hline 3DOm-i LTA & 531 & 228 & 0.15 & 0.48 \\
\hline 3DOm-i BEA & 653 & 254 & 0.19 & 0.50 \\
\hline MCM-22 & 432 & 66 & 0.19 & 0.31 \\
\hline $300 \mathrm{~nm} \mathrm{MFI}$ & 438 & 134 & 0.13 & 0.28 \\
\hline ITQ-2 & 674 & 345 & 0.20 & 0.50 \\
\hline Al-MCM-41 & 824 & 22 & N.A. & 0.78 \\
\hline
\end{tabular}

[a] Calculated using the $t$-plot method. [b] Calculated from the adsorption branch at $P / P_{0}=0.95$.

the 3DOm-i zeolite MFI, LTA, and BEA are 477, 531, and $653 \mathrm{~m}^{2} \mathrm{~g}^{-1}$, respectively. The external surface area $\left(S_{\text {ext }}\right)$ is $228-$ $254 \mathrm{~m}^{2} \mathrm{~g}^{-1}$, which indicates that a large external surface area is created in the hierarchical zeolites. The micropore volumes calculated from the $t$-plot method are 0.14, 0.15, and $0.19 \mathrm{~cm}^{3} \mathrm{~g}^{-1}$, respectively, which implies the high crystallinity of the 3DOm-i zeolite catalysts. However, MCM-22 only shows characteristic micropore filling $\left(P / P_{0}<0.05\right)$ without any feature of mesoporosity. The ITQ-2 sample shows a similar microporosity to MCM-22 but a significantly larger mesopore volume and external surface area (Table 2), which again indicates that the micropore structure of ITQ-2 was retained well during the delamination process. The external surface area of $\mathrm{MCM}-22$ is $66 \mathrm{~m}^{2} \mathrm{~g}^{-1}$, which is much smaller than that of the 3DOm-i zeolites. It can be concluded that all the 3DOm-i samples have both highly crystalline microporous structures associated with zeolite domains and mesoporous structures inherited from the 3DOm carbon template. However, the MCM-22 sample mainly exhibits microporous structures because of the condensation of the layered MCM-22 precursors during calcination. ${ }^{[4 b, c]}$ 


\section{Acid site distribution}

The distribution of Brønsted acid sites in the hierarchical zeolites was investigated by using temperature-programmed desorption (TPD). Isopropylamine (IPA), 2,4,6-collidine (CLD), and triphenylphosphine (TPP) with different molecular sizes served as probe molecules to access the total acid sites (IPA-TPD) and acid sites on the external surface of the zeolite catalysts (CLDTPD or TPP-TPD).

IPA-TPD was used to determine the total number of Brønsted acid sites in each catalyst, which is supported by the fact that adsorption occurs with a 1:1 stoichiometry of IPA over the Brønsted acid sites in zeolite catalysts. ${ }^{[15]}$ The Si/Al ratio and Al concentration of the zeolite catalysts determined by elemental analysis (ICP-OES) are given in Table 3. The number of accessible Brønsted acid sites obtained from the IPA-TPD experiments is similar to the amount of $\mathrm{Al}$ in $3 \mathrm{DOm}-\mathrm{i} \mathrm{ZSM}-5$, which indicates that most of the $\mathrm{Al}$ atoms are incorporated within the framework of these zeolite catalysts to lead to Brønsted acidity. This result is consistent with that of FTIR spectroscopy using pyridine as the probe molecule (Figure S5). 3DOm-i BEA and MCM-22 show lower Brønsted acid concentrations than the amount of $\mathrm{Al}$ in the zeolites, which indicates the presence of extra-framework Al. Interestingly, the total Brønsted acid sites in 3DOm-i LTA measured by using IPA-TPD is significantly lower than the amount of $\mathrm{Al}$ in this sample. This is because IPA cannot diffuse into the $8 \mathrm{MR}$ in the LTA structure and access the acid sites located on the internal surface at the adsorption temperature of $393 \mathrm{~K}$. The observation is consistent with the previous study of Pereira and Gorte. ${ }^{[15 b]}$ The acid site concentration for 3DOm-i LTA reported in Table 3 is, thus, only related to the accessible acid sites on the external surface of the catalyst.

To evaluate the acid sites on the external surface of the zeolite samples, a bulky amine molecule, CLD, was used for 3DOm-i MFI, MCM-22, and ITQ-2 in the TPD measurements. ${ }^{[16]}$ As CLD, which has a kinetic diameter around $0.74 \mathrm{~nm}$, can diffuse easily into BEA micropores, ${ }^{[17]}$ an even larger base mole-

\begin{tabular}{|c|c|c|c|c|c|c|}
\hline \multirow[t]{2}{*}{ Catalyst } & \multirow[t]{2}{*}{$\mathrm{Si} / \mathrm{Al}$} & \multicolumn{3}{|c|}{ Acid concentration $\left[\mathrm{mmolg}^{-1}\right]$} & \multicolumn{2}{|r|}{$f_{\mathrm{B}, \mathrm{ext}}$} \\
\hline & & $\begin{array}{l}\text { Total }^{[\mathrm{a}]} \\
\text { (theoretical) }\end{array}$ & Total $^{[b]}$ & $\begin{array}{l}\text { External } \\
\text { surface }^{[c]}\end{array}$ & $\begin{array}{l}\text { (actual) } \\
{[\%]^{[d]}}\end{array}$ & $\begin{array}{l}\text { (theoretical) } \\
{[\%]^{[\mathrm{e}]}}\end{array}$ \\
\hline 3DOm-i MFI & 151 & 0.110 & 0.113 & 0.016 & 14.2 & 9.6 \\
\hline 3DOm-i LTA & 2.2 & 5.208 & 0.140 & 0.140 & 100 & 6.5 \\
\hline 3DOm-i BEA & 34 & 0.476 & 0.370 & 0.037 & 10.0 & 9.4 \\
\hline MCM-22 & 45 & 0.362 & 0.346 & 0.022 & 6.4 & 9.8 \\
\hline $300 \mathrm{~nm} \mathrm{MFI}$ & 48 & 0.340 & 0.338 & 0.015 & 2.4 & 7.3 \\
\hline ITQ-2 & 50 & 0.327 & 0.325 & 0.179 & 55.1 & 100 \\
\hline Al-MCM-41 & 204 & 0.407 & 0.081 & N.A. & N.A. & N.A. \\
\hline
\end{tabular}

[a] Number of total Brønsted acid sites calculated based on Si/Al ratio. [b] Number of total Brønsted acid sites determined by IPA-TPD. [c] Number of external Brønsted acid sites determined from CLD-TPD for 3DOm-i MFI, 3DOm-i LTA, MCM-22, and ITQ-2 and TPP-TPD for 3DOm-i BEA. [d] Fraction of external Brønsted acid sites calculated by (number of Brønsted acid sites by CLD-TPD or TPP-TPD/number of Brønsted acid sites by IPA-TPD). For 3DOm-i LTA, Brønsted acid sites detected by IPA are assigned to the acid sites on the external surface. [e] Calculated based on crystal shape and size and assuming a random distribution of Brønsted acid sites. cule, TPP, was used for 3DOm-i BEA. The acid site concentration on the external surface and the ratio of external acid sites to the total acid site measured by CLD-TPD or TPP-TPD are listed in Table 3. The fraction of external Brønsted acid sites accessible to the bulky base molecules in 3DOm-i MFI and 3DOm-i BEA is 14.2 and $10.1 \%$, respectively, which is approximately twice as high as that of MCM-22 (6.4\%). The higher distribution of the Brønsted acid sites on the external surface of the 3DOm-i zeolites is mainly because of the hierarchical structures of the zeolite catalysts. The fraction of external Brønsted acid sites accessible to CLD on ITQ-2 (55.1\%) is much larger than that on MCM-22 because of the delaminated structure of ITQ-2. The values are also consistent with the theoretical values calculated from the crystal morphologies with the assumption of a random distribution of Brønsted acid sites. The results support that the hierarchical structures created by the hard-templating method and delamination method can significantly improve the acid site distributions on the external surface of the zeolite catalysts.

\section{Catalytic activity}

3DOm-i zeolites with imprinted mesoporous structures and a large number of accessible Brønsted acid sites exhibit remarkable benefits for the catalytic reactions in which diffusion constraints and/or adsorption of reactant molecules onto the active acid sites are the main concerns. In this study, the liquid-phase catalytic conversion of benzyl alcohol in mesitylene was used to evaluate the catalytic performance of the hierarchical zeolite catalysts. Previous studies showed that two parallel reactions, the self-etherification of benzyl alcohol and the alkylation of mesitylene with benzyl alcohol, can be catalyzed by the Brønsted acid sites of zeolite catalysts. ${ }^{[8,18]}$ The products of the two reactions are dibenzyl ether (DE) and 1,3,5-trimethyl-2-benzylbenzene (TMBB), respectively (Scheme 1). It has been revealed that the alkylation of mesitylene with benzyl alcohol is catalyzed exclusively by the acid sites on the external surface of MFI zeolites because of the steric resistance of the bulky reactant and product, mesitylene and TMBB. The poisoning of the external surface of the MFI zeolite catalysts with bulky amines such as 2,6-di-tert-butylpyridine (DTBP) can eliminate the production of TMBB completely. However, the production of DE from the self-etherification of benzyl alcohol can be catalyzed by the acid sites located on both the external and the internal surface of MFI zeolite catalysts. As a result of the relatively large molecular size of benzyl alcohol, diffusion constraints have been observed for the reaction in MFI zeolites, and a diffusion-reaction model has been developed to interpret the reaction results. ${ }^{[18 a, 19]} \mathrm{A}$ study of the liquid-phase conversion of benzyl alcohol in mesitylene over the hierarchical zeolite catalysts provides critical insights into the catalytic activity and selectivity of the hierarchical zeolite catalysts.

In this study, the conversion of benzyl alcohol in mesitylene over various zeolite catalysts was per- 


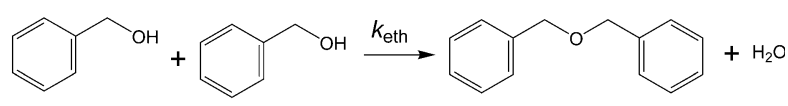

$b$

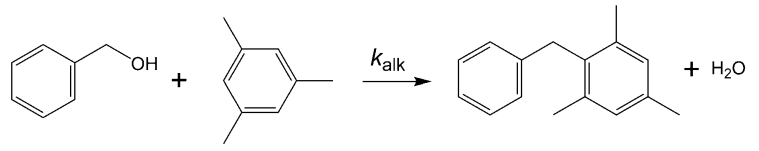

Scheme 1. Two reaction pathways of benzyl alcohol in mesitylene, a) benzyl alcohol self-etherification and b) alkylation of mesitylene with benzyl alcohol.

formed to evaluate the effects of mesoporous and microporous structures on the catalytic performance. The conversions of benzyl alcohol in mesitylene over various zeolite catalysts at $343 \mathrm{~K}$ are shown in Figure 4. Without poisoning the acid sites on the external surface, 3DOm-i BEA showed a higher conversion of benzyl alcohol than the other 3DOm-i zeolite catalysts because the large $12 \mathrm{MR}$ and mesoporous structures led to enhanced mass transport for the bulky reactants and products (Figure 4a). ITQ-2 and AI-MCM-41 also exhibited a high conversion because of the ease of access to the acid sites located on the external surface. As expected, 3DOm-i MFI exhibited higher conversions than $300 \mathrm{~nm}$ MFI because of the imprinted mesoporous structures. 3DOm-i LTA and MCM-22 can also catalyze the reaction but with much lower conversions, mainly because of their small microporous structures that limit the reaction that occurs exclusively on the external surface of the catalysts. These results suggest that the presence of mesopores and large micropores (e.g., $12 \mathrm{MR}$ ) can reduce the diffusion limitation in the alkylation of mesitylene with benzyl alcohol and etherification of benzyl alcohol significantly. For this reason, 3DOm-i BEA exhibited superior catalytic performances for the reactions.

In contrast to mesoporous Al-MCM-41, the hierarchical zeolites with different microporosities offer an additional parameter to control the product selectivity in the reactions. The selectivities to TMBB and DE were calculated at benzyl alcohol conversions below $10 \%$ (Table 4). Remarkably, a distinct difference in the product selectivity was observed between the zeolite catalysts and mesoporous Al-MCM-41. For 3DOm-i BEA and 3DOm-i MFI, the main product was $D E$ from the etherification reaction, whereas the alkylated product, TMBB, was predominant for 3DOm-i LTA, MCM-22, ITQ-2, and AlMCM-41. The difference in product selectivity arises from the small pore structure of 3DOm-i LTA, MCM22, and ITQ-2, which limited most reactions that occur on the external surface to favor the formation of alkylated product. In addition, the external surface structure and terminal group concentration of the four zeolites are also significantly different, which might also lead to different product selectivity as proposed previously. ${ }^{[11 b, 20]}$

The product selectivity can be further controlled by tailoring the reactions selectively over the external surface or internal surface of the hierarchical zeolites. It has been reported that poisoning the acid
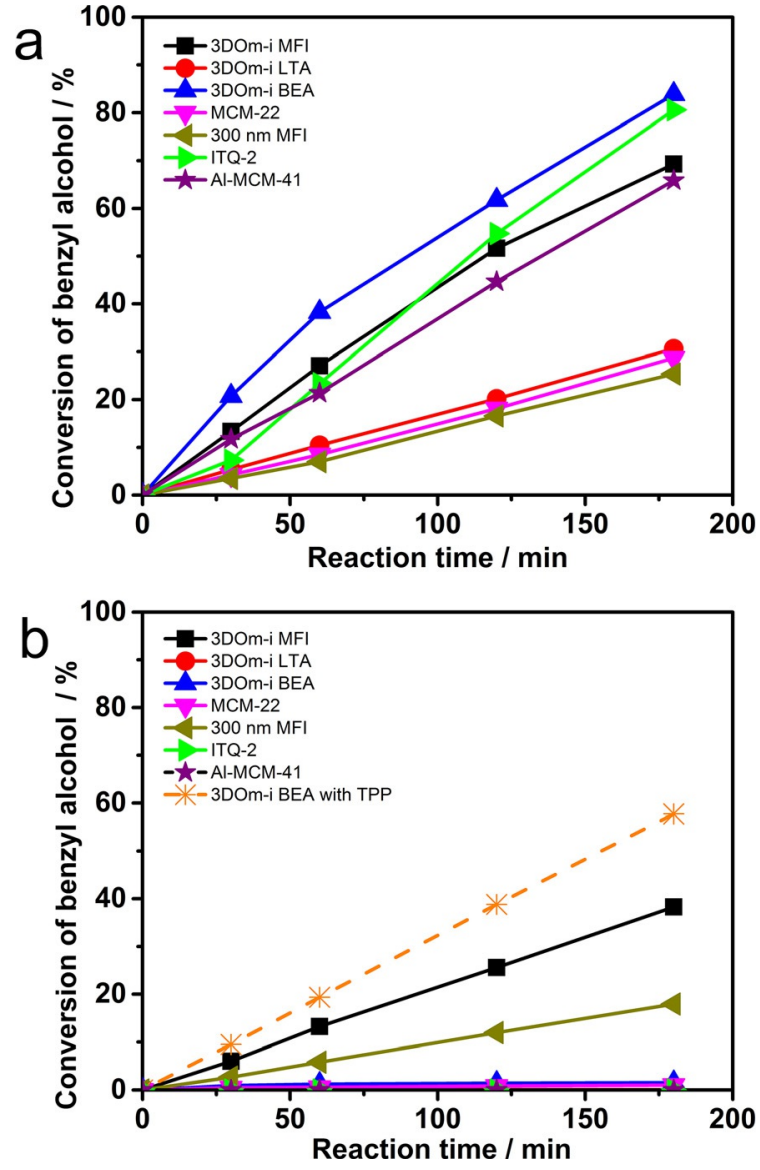

Figure 4. Catalytic conversion of benzyl alcohol in mesitylene over various zeolite catalysts. a) Mesitylene alkylation with benzyl alcohol (without DTBP) and b) benzyl alcohol self-etherification (with DTBP or TPP).

sites on the external surface of zeolite catalysts with bulky base molecules can prevent the formation of alkylated product completely in the reactions. ${ }^{[8]}$ In the presence of DTBP, the conversion of benzyl alcohol was decreased significantly for
Table 4. Catalytic performance of the microporous and mesoporous catalysts for the liquid-phase catalytic reactions of benzyl alcohol in mesitylene.

\begin{tabular}{|c|c|c|c|c|}
\hline \multirow[t]{3}{*}{ Catalyst } & \multicolumn{3}{|c|}{ Reaction rate constant ${ }^{[\mathrm{a}]}$} & \multirow{3}{*}{$\begin{array}{l}\text { Selectivity } \\
\left(\alpha=2 C_{\mathrm{E}} / C_{\mathrm{C}}\right)\end{array}$} \\
\hline & \multirow{2}{*}{$\begin{array}{l}\text { Alkylation }{ }^{[\mathrm{b}]} \\
{\left[10^{-3} \mathrm{~s}^{-1}\left(\mathrm{~mol} \mathrm{H}^{+}\right)^{-1}\right]} \\
k_{\text {alk,ext }}\end{array}$} & \multicolumn{2}{|c|}{$\begin{array}{l}\text { Self-etherification }{ }^{[\mathrm{c}]} \\
{\left[10^{-6} \mathrm{Ls}^{-1}\left(\mathrm{~mol} \mathrm{H}^{+}\right)^{-1}\right]}\end{array}$} & \\
\hline & & $k_{\text {alk,int }}{ }^{\text {[e] }}$ & $k_{\text {eth,app,int }}^{[\mathrm{e}]}$ & \\
\hline 3DOm-i MFI & 934.1 & 0 & 764.9 & 5.3 \\
\hline 3DOm-i LTA & 722.3 & 0 & 0 & 0.4 \\
\hline 3DOm-i BEA & 1882.0 & 0 & 0 & 6.4 \\
\hline 3DOm-i BEA ${ }^{[f]}$ & N.A. & 0 & $2140.9^{[f]}$ & N.A. \\
\hline MCM-22 & 9663.3 & 0 & 0 & 0.7 \\
\hline 300 nm MFI & 634.9 & 0 & 94.7 & 12.2 \\
\hline ITQ-2 & 6526.8 & 0 & 0 & 0.6 \\
\hline Al-MCM-41 & 1543.2 & 0 & 0 & 0.3 \\
\hline
\end{tabular}

[a] Rate parameters determined by least-squares regression. [b] Without DTBP. [c] With DTBP. [d] Normalized to the number of external Brønsted acid sites determined by CLD-TPD or TPP-TPD. [e] Normalized to the number of internal Brønsted acid sites (internal acid sites = total acid sites-external acid sites). [f] Calculated if TPP was added in the reaction. 
3DOm-i MFI and almost completely eliminated for 3DOmi BEA, 3DOm-i LTA, MCM-22, ITQ-2, and Al-MCM-41 (Figure 4b). The detectable product from 3DOm-i MFI was DE only. No alkylated product, TMBB, was observed in the reaction. The results suggest clearly that alkylated product was catalyzed by the acid sites on the external surface of the zeolite catalysts. The formation of DE can be catalyzed by the acid sites on the external and internal surface of MFI zeolite simultaneously. However, because of the small pore size of LTA and MCM-22, the acid sites on the internal surface cannot be utilized for $D E$ production. Surprisingly, the catalytic activity of 3DOm-i BEA was also almost completely prohibited in the presence of DTBP. This is likely because DTBP can poison the acid sites on both the external and internal surface of the large-pore zeolite. To confirm the observation, instead of DTBP, TPP was added in the reaction to poison the acid sites on the external surface of BEA zeolite selectively. As expected, TPP was adsorbed exclusively on the external surface of $3 D O m-i$ BEA, which led to a much higher conversion of benzyl alcohol than in the case of DTBP. A single product (DE) was observed in the reaction in the presence of TPP, which indicates that the $12 \mathrm{MR}$ in BEA zeolite cannot allow the formation of alkylated products in their micropores although the pore size is larger than that of MFI. The results show clearly the unique catalytic activity of hierarchical zeolites for the selective production of DE from the self-etherification reaction. 3DOm-i BEA achieved the highest conversion of benzyl alcohol to DE on poisoning the acid sites on the external surface because of the $12 \mathrm{MR}$ structure and interconnected mesopores. 3DOm-i MFI also produced DE selectively over the acid sites located within the zeolite but with a lower activity compared to 3DOm-i BEA because of diffusion limitations (10 MR vs. $12 \mathrm{MR}$ ). Neither small-pore zeolites (e.g., LTA) nor mesoporous Al-MCM-41 can produce DE selectively from the reaction because of the lack of the control of the acid site distributions for exclusive access by benzyl alcohol.

To evaluate the catalytic activity of the acid sites within the hierarchical zeolites, the reaction rate constants for the conversion of benzyl alcohol in mesitylene over the external and internal surface of the hierarchical zeolites were calculated. The conversion data obtained with and without the addition of bulky base molecules fitted well to a pseudo-first-order reaction model (Supporting Information). This allows us to determine the alkylation reaction and etherification reaction rate constants, respectively. First, the alkylation reaction rate constant $k_{\text {alk,ext }}$ was calculated from the experimental data using Equation (S7) and is shown in Table 4 and Figure 5. Although the alkylation reaction only occurred on the external surface, the rate constant varied significantly with the zeolite structures. Interestingly, the difference was as large as one order of magnitude from 0.634 to $9.663 \mathrm{~s}^{-1}$ for MCM-22 and $300 \mathrm{~nm}$ $\mathrm{MFI}$, respectively. The results suggest that it is possible to tailor the catalytic activity of zeolite catalysts by fabricating their external surface properties. It is known that several parameters, which include adsorption properties, acid strength, steric constraints, and Al distribution, can affect the catalytic activity of zeolite catalysts. In particular for the alkylation reaction on the external surface, the reaction rate constant cannot be associat-
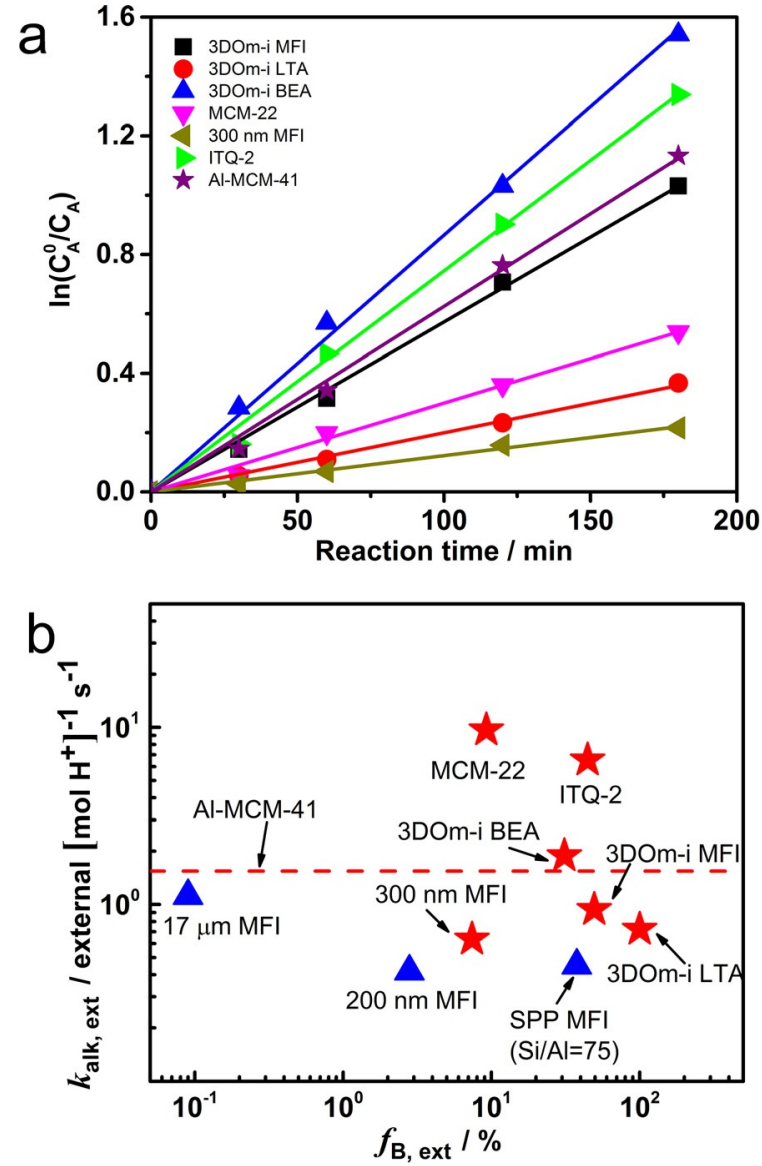

Figure 5. a) Plots for the determination of the rate constants of benzyl alcohol conversion in the absence of DTBP. (The selectivity over each catalysts can be found from Table 4. Reaction conditions: total amount of catalysts used are 3DOm-i MFI: $0.1 \mathrm{~g}$, 3DOm-i LTA: $0.2294 \mathrm{~g}, 3 D O m-i$ BEA: $0.0692 \mathrm{~g}$, MCM-22: 0.0948 g, $300 \mathrm{~nm}$ MFI: $0.0970 \mathrm{~g}$, ITQ-2: 0.1009 g, Al-MCM-41: $0.4049 \mathrm{~g}$. The slope of each plot is the apparent rate constant $\left.k_{\mathrm{alk}, \mathrm{app}}\right)$. b) Reaction rate constant for mesitylene alkylation by benzyl alcohol $\left(k_{\text {alk,ext }}\right)$ for various zeolite catalysts. (The data for $17 \mu \mathrm{m} \mathrm{MFI,} 200 \mathrm{~nm} \mathrm{MFI}$, and SPP MFI $(\mathrm{Si} / \mathrm{Al}=75)$ are from Ref. [8].)

ed directly with a single parameter of the zeolite catalysts, because of the unknown acid site distribution, terminal structure, and defects in the structure. Therefore, based on the reaction results, it cannot be concluded if the acid site strength of a certain zeolite is distinctly different from that of the others.

The self-etherification reaction rate constants within the micropores of different hierarchical zeolites are shown in Figure 6 and Table 4. 3DOm-i BEA exhibited the highest rate constant because of the enhanced mass transport and the large $12 \mathrm{MR}$ structure. 3DOm-i MFI exhibited a lower rate constant than 3DOm-i BEA but a higher value than $300 \mathrm{~nm} \mathrm{MFI}$, which indicates the presence of diffusion constraints in MFI zeolites. The rate constants of 3DOm-i LTA, MCM-22, ITQ-2, and AI-MCM-41 are close to zero because DTBP can block the accessible acid sites for the self-etherification reaction completely. For the same reason, so is that of 3DOm-i BEA in the presence of TPP. The reaction rate constant for 3DOm-i BEA is similar to that of self-pillared pentasil (SPP) zeolite with a diffusion length of only approximately $2 \mathrm{~nm}$ in which the diffusion limitation was 

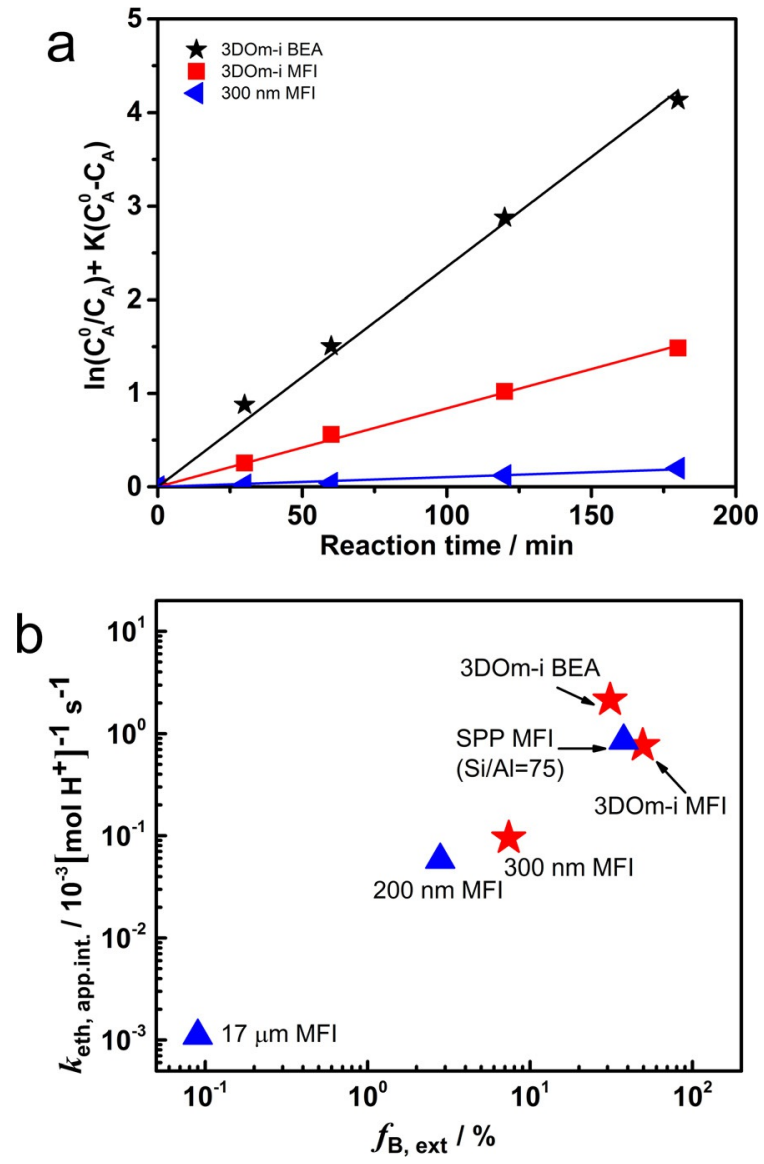

Figure 6. a) Plots for the determination of the apparent initial etherification rate constants of benzyl alcohol conversion inside the micropores in the presence of DTBP or TPP over different zeolite catalysts. (Reaction conditions: total amount of catalysts used are 3DOm-i BEA: $0.069 \mathrm{~g}, 3 \mathrm{DOm}-\mathrm{i} \mathrm{MFI}$ : $0.1 \mathrm{~g}, 300 \mathrm{~nm}$ MFI: $0.097 \mathrm{~g}$. The slope of each plot is $k_{\text {eth.appint, }}$ which can be determined from Equation S2). b) Apparent internal self-etherification reaction rate constant $k_{\text {eth,app,int }}$ for various zeolite catalysts. (The data for $17 \mu \mathrm{m}$ $\mathrm{MFI}, 200 \mathrm{~nm} \mathrm{MFI}$, and SPP MFI (Si/Al=75) are from Ref. [8].)

not observed. The similar reaction rate constant suggests that the self-etherification of benzyl alcohol in the 3DOm-i BEA is not constrained by diffusion limitations. The intrinsic reaction rate of self-etherification of the BEA and MFI zeolites should not be significantly different.

\section{Conclusions}

Hierarchical zeolites with ordered mesoporous structures and various frameworks were synthesized using a hard-templating method. The liquid-phase catalytic reactions of benzyl alcohol in mesitylene were used as model reactions to evaluate the catalytic activity of the acid sites on both the external and internal surfaces. Two parallel reactions occurred over these zeolite catalysts. The self-etherification of benzyl alcohol can be catalyzed by acid sites on both the external and internal surface of MFI and BEA zeolites. The ten- and eight-memberedring windows of MWW and LTA hinder the etherification reaction of benzyl alcohol. The alkylation of mesitylene with benzyl alcohol was catalyzed exclusively by the acid sites on the external surface of MFI, BEA, LTA, MCM-22, ITQ-2, and AI-MCM-41.
The introduction of mesoporosity in the zeolite structures can improve their catalytic performances remarkably by facilitating the accessibility of the acid sites within the micropores. After the selective poisoning of the acid sites on the external surface by bulky base molecules, the catalytic activity of the acid sites on the internal surface of zeolite catalysts can be evaluated. Among the hierarchical zeolites, 3DOm-i BEA exhibited the highest activity for the etherification of benzyl alcohol, which indicates that the large micropore and hierarchical structure can reduce the diffusion limitation for the reaction effectively. Interestingly, without the poisoning of the acid sites on the external surface, distinct product selectivity was achieved with the zeolite catalysts. Namely, 3DOm-i MFI, 3DOm-i BEA, and $300 \mathrm{~nm}$ MFI prefer the etherification of benzyl alcohol, whereas 3DOm-i LTA, MCM-22, ITQ-2, and AI-MCM-41 favor the formation of the alkylated product from benzyl alcohol and mesitylene. This is likely because of the different external surface structures of the zeolite catalysts and the corresponding adsorption properties and acid strength. This study suggested that the liquid-phase catalytic reactions of benzyl alcohol in mesitylene over hierarchical zeolite catalysts can provide critical insights into the catalytic performance of hierarchical zeolites. Furthermore, the presence of a mesoporous structure and a large external surface area on hierarchical zeolites not only enhances the catalytic activities but also can change the selectivities of the zeolite catalysts.

\section{Experimental Section}

\section{Catalyst preparation}

3DOm-i zeolites with MFI, BEA, and LTA frameworks were synthesized according to previous reports. ${ }^{[9,21]}$ The detailed synthesis procedure is available in the Supporting Information. For comparison, MFI zeolite nanocrystals with a particle size of $300 \mathrm{~nm}$, MWW zeolites (MCM-22 and ITQ-2), and mesoporous AI-MCM-41 were prepared according to methods published previously. ${ }^{[4,22]}$ lon exchange of all catalysts was performed with a $1 \mathrm{~m}$ aqueous solution of $\mathrm{NH}_{4} \mathrm{NO}_{3}$ at $353 \mathrm{~K}$ for $2 \mathrm{~h}$ and repeated three times. The final products were converted to the proton form by calcination at $823 \mathrm{~K}$ for $8 \mathrm{~h}$ with a ramping rate of $1 \mathrm{Kmin}^{-1}$ in a dry air flow.

\section{Characterization}

The XRD measurements were performed by using an X'pert Powder (PANalytical) diffractometer using $\mathrm{CuK}_{\alpha}$ radiation $(\lambda=$ $0.15418 \mathrm{~nm}$ ) with a Ni filter from $2 \theta=4$ to $40^{\circ}$ with a step size of $0.04^{\circ}$. Small-angle X-ray scattering (SAXS) patterns were collected by using a Molecular Metrology SAXS line using a $\mathrm{CuK}_{\alpha}$ radiation with a sample-to-detector distance of $1.48 \mathrm{~m}$. SEM images were recorded by using a FEl-Magellan 400 microscope, equipped with a field-emission gun operated at $3.0 \mathrm{kV}$. The samples were coated with Pt by sputtering at $60 \mathrm{~mA}$ for $60 \mathrm{~s}$ by using a Sputter Coater 208. TEM images were taken by using a JEOL 2000FX microscope operated at $100 \mathrm{kV}$. The samples were first dispersed in ethanol with sonication, and then placed on a carbon-coated copper grid followed by evaporation at ambient conditions.

$\mathrm{N}_{2}$ physisorption isotherms at $77 \mathrm{~K}$ were measured by using a Quantachrome gas adsorption analyzer (Autosorb ${ }^{\circledR} \mathrm{iQ}_{2}$ ). Before 
the analysis, all catalysts were degassed at $573 \mathrm{~K}$ for $12 \mathrm{~h}$. The specific surface area was calculated using BET equation based on the adsorption branch curve obtained at $P / P_{0}=0.05-0.3$. The pore size distributions were obtained from the entire adsorption branch from on a NLDFT method, which describes $\mathrm{N}_{2}$ adsorbed onto silica with cylindrical pores at $77 \mathrm{~K}$ (Versawin ${ }^{\mathrm{TM}}$ 1.0, Quantachrome).

TPD measurements were performed by using a thermogravimetric analysis instrument (TA ${ }^{\circledR}$ SDT Q600) to investigate the number of Brønsted acid sites of the catalysts following the method developed by Gorte et al. ${ }^{[14 b, 23]}$ Before the analysis, the catalysts $(10.0-$ $15.0 \mathrm{mg}$ ) were pretreated at $773 \mathrm{~K}$ for $1 \mathrm{~h}$ in a He flow to clean the catalyst surface. After the samples were cooled to $393 \mathrm{~K}$, an IPA/He gas mixture was passed through the sample at $100 \mathrm{~mL} \mathrm{~min}^{-1}$. After the adsorption of IPA reached equilibrium on the catalysts, the temperature was increased from 393 to $1023 \mathrm{~K}$ with a ramping rate of $10 \mathrm{Kmin}^{-1}$. The decomposition of IPA into ammonia and propene on the Brønsted acid sites of zeolite catalysts that occurs between 575 and $650 \mathrm{~K}$ was used to calculate the total amount of Brønsted acid sites of the catalysts. CLD, which is too bulky to diffuse into MFI micropores, was used to quantify acid sites on the external surface of 3DOm-i ZSM-5 ${ }^{[8]}$ and MCM-22. ${ }^{[24]}$ A typical CLDTPD experiment was conducted by using the same thermogravimetric analysis instrument as IPA-TPD, except CLD was used as the adsorbate. A larger basic molecule, TPP, was used to evaluate the acid sites on the external surface of BEA zeolite as suggested previously. ${ }^{[11 b, 20 c, 25]} 3 \mathrm{DOm}$-i BEA was first calcined at $823 \mathrm{~K}$ for $8 \mathrm{~h}$ in an air flow to remove the adsorbed impurities. $\mathrm{CH}_{2} \mathrm{Cl}_{2}$ solution $(1 \mathrm{~mL})$ that contained TPP $\left(10^{-7} \mathrm{M}\right)$ was added to the calcined $\mathrm{BEA}$ zeolite. The sample was washed with an excess amount of $\mathrm{CH}_{2} \mathrm{Cl}_{2}$ to remove the weakly adsorbed TPP. Finally, the sample was dried in an oven at $393 \mathrm{~K}$ overnight to remove $\mathrm{CH}_{2} \mathrm{Cl}_{2}$. The subsequent procedure was the same as described above for the IPA-TPD and CLDTPD measurements. For the case of AI-MCM-41, IPA-TPD was used to measure the amount of acid sites on the surface.

\section{Catalytic reaction}

The liquid-phase catalytic conversion of benzyl alcohol in mesitylene was chosen as a model reaction to investigate the catalytic performance of 3DOm-i zeolites and other conventional zeolites with a large external surface area. The reaction was performed in a three-necked round-bottom flask $(100 \mathrm{~mL})$ equipped with a reflux condenser under atmospheric pressure. The temperature of the reflux condenser was kept at $270 \mathrm{~K}$. The flask was heated in a temperature-controlled oil bath ( $343 \mathrm{~K})$ with magnetic stirring at $500 \mathrm{rpm}$. Before the reactions, all catalysts were activated in a muffle furnace at $823 \mathrm{~K}$ for $4 \mathrm{~h}$ under a dry air flow $\left(100 \mathrm{~mL} \mathrm{~min}{ }^{-1}\right)$. Typically, $0.15 \mathrm{~g}$ of 3DOm-i BEA, which corresponds to $7.04 \times 10^{-5} \mathrm{~mol}$ of Brønsted acid sites, was added to the flask that contained mesitylene $(15 \mathrm{~mL}, 98 \%$, Aldrich). The amount of 3DOm-i MFI, 3DOm-i LTA, MCM-22, ITQ-2, and Al-MCM-41 used in the reactions was adjusted to keep the amount of Brønsted acid in the reactor the same as the case of 3DOm-i BEA. The reaction mixture was maintained for $1 \mathrm{~h}$ at $343 \mathrm{~K}$ with stirring before the addition of benzyl alcohol $(0.25 \mathrm{~mL}$, ACS reagent, $\geq 99.0 \%$, Aldrich). The reaction time was started at the moment when benzyl alcohol was added. Samples were collected periodically and analyzed by using GC (Agilent HP-6890 GC) equipped with a methylsiloxane capillary column (HP-1, $50.0 \mathrm{~m} \times 20 \mu \mathrm{m} \times 0.52 \mu \mathrm{m})$ connected to a flame ionization detector.

According to previous studies, DTBP ( $\geq 97 \%$, Aldrich) can be used to poison the Brønsted acid sites on the external surface of zeolite catalysts with MFI and MWW structures selectively without changing the performance of the active sites located on the internal surface significantly. ${ }^{[18 a, 19]}$ To study the reactions catalyzed only by the acid sites on the internal surface of the 3DOm-i zeolite catalysts, an excess amount of DTBP was added in the reactor (five times molar excess of DTBP) before the reaction. The mixture was then heated to reflux for $2.5 \mathrm{~h}$ at $343 \mathrm{~K}$ with stirring to make sure that DTBP was adsorbed on the acid sites located on the external surface of the zeolite catalysts. Benzyl alcohol $(0.25 \mathrm{~mL})$ was then added to the mixture. In the case of 3DOm-i BEA, TPP was used to poison the external acid sites of 3DOm-i BEA as DTBP can diffuse into the micropores of BEA zeolites. Liquid samples were collected and analyzed using the same method described above. A blank test was also conducted under the same conditions without the addition of catalysts. The result showed that there was no conversion of benzyl alcohol after $3 \mathrm{~h}$ in the absence of zeolite catalysts.

\section{Acknowledgements}

This work was supported by the Catalysis Center for Energy Innovation, an Energy Frontier Research Center funded by the US Dept. of Energy, Office of Science, and Office of Basic Energy Sciences under award number DE-SC0001004.

Keywords: acidity - alcohols · alkylation - mesoporous
materials - zeolites

[1] a) A. Corma, Chem. Rev. 1997, 97, 2373-2420; b) J. Pérez-Ramírez, C. H. Christensen, K. Egeblad, J. C. Groen, Chem. Soc. Rev. 2008, 37, 2530 2542; c) W. J. Roth, P. Nachtigall, R. E. Morris, J. Čejka, Chem. Rev. 2014, $114,4807-4837$.

[2] a) M. E. Davis, Nature 2002, 417, 813-821; b) B. Smit, T. L. M. Maesen, Chem. Rev. 2008, 108, 4125-4184.

[3] a) C. M. A. Parlett, K. Wilson, A. F. Lee, Chem. Soc. Rev. 2013, 42, 3876 3893; b) K. Egeblad, C. H. Christensen, M. Kustova, C. H. Christensen, Chem. Mater. 2008, 20, 946-960; c) M. Hartmann, Angew. Chem. Int. Ed. 2004, 43, 5880-5882; Angew. Chem. 2004, 116, 6004-6006; d) Y. S. Tao, H. Kanoh, L. Abrams, K. Kaneko, Chem. Rev. 2006, 106, 896-910; e) J. Cejka, S. Mintova, Catal. Rev. Sci. Eng. 2007, 49, 457-509.

[4] a) K. Na, M. Choi, W. Park, Y. Sakamoto, O. Terasaki, R. Ryoo, J. Am. Chem. Soc. 2010, 132, 4169-4177; b) A. Corma, V. Fornes, S. B. Pergher, T. L. M. Maesen, J. G. Buglass, Nature 1998, 396, 353-356; c) S. Maheshwari, E. Jordan, S. Kumar, F. S. Bates, R. L. Penn, D. F. Shantz, M. Tsapatsis, J. Am. Chem. Soc. 2008, 130, 1507-1516; d) I. Ogino, E. A. Eilertsen, S.-J. Hwang, T. Rea, D. Xie, X. Ouyang, S. I. Zones, A. Katz, Chem. Mater. 2013, 25, 1502-1509.

[5] J. C. Groen, J. Pérez-Ramírez, in Novel Concepts in Catalysis and Chemical Reactors (Eds.: A. Cybulski, J. A. Moulijn, A. Stankiewicz), Wiley-VCH, Weinheim, 2010, pp. $31-50$.

[6] a) M. Choi, K. Na, J. Kim, Y. Sakamoto, O. Terasaki, R. Ryoo, Nature 2009, 461, 246-249; b) W. Fan, M. A. Snyder, S. Kumar, P.-S. Lee, W. C. Yoo, A. V. McCormick, R. Lee Penn, A. Stein, M. Tsapatsis, Nat. Mater. 2008, 7, 984-991.

[7] K. Na, C. Jo, J. Kim, K. Cho, J. Jung, Y. Seo, R. J. Messinger, B. F. Chmelka, R. Ryoo, Science 2011, 333, 328-332.

[8] X. Zhang, D. Liu, D. Xu, S. Asahina, K. A. Cychosz, K. V. Agrawal, Y. Al Wahedi, A. Bhan, S. Al Hashimi, O. Terasaki, M. Thommes, M. Tsapatsis, Science 2012, 336, 1684-1687.

[9] H. Chen, J. Wydra, X. Zhang, P.-S. Lee, Z. Wang, W. Fan, M. Tsapatsis, J. Am. Chem. Soc. 2011, 133, 12390-12393.

[10] M. S. Holm, E. Taarning, K. Egeblad, C. H. Christensen, Catal. Today 2011, $168,3-16$.

[11] a) J.-C. Kim, K. Cho, R. Ryoo, Appl. Catal. A 2014, 470, 420-426; b) W. Kim, J.-C. Kim, J. Kim, Y. Seo, R. Ryoo, ACS Catal. 2013, 3, 192-195; c) K. Kim, R. Ryoo, H.-D. Jang, M. Choi, J. Catal. 2012, 288, 115-123. 
[12] a) Ch. Baerlocher, L. B. McCusker, D. H. Olson, Atlas of Zeolite Framework Types, $6^{\text {th }}$ Ed., Elsevier, Amsterdam, 2007; b) W. M. Meier, D. H. Olson, Zeolites 1992, 12, 16-185.

[13] A. Corma, V. Fornés, J. M. Guil, S. Pergher, T. L. M. Maesen, J. G. Buglass, Microporous Mesoporous Mater. 2000, 38, 301 - 309.

[14] A. Corma, V. Fornes, J. Martinez-Triguero, S. B. Pergher, J. Catal. 1999 186, 57-63.

[15] a) O. Kresnawahjuesa, R. J. Gorte, D. de Oliveira, L. Y. Lau, Catal. Lett. 2002, 82, 155-160; b) C. Pereira, R. J. Gorte, Appl. Catal. A 1992, 90, $145-157$.

[16] a) M. Niwa, N. Katada, Chem. Rec. 2013, 13, 432-455; b) A. Corma, V. Fornés, L. Forni, F. Márquez, J. Martínez-Triguero, D. Moscotti, J. Catal. 1998, 179, $451-458$.

[17] F. Thibault-Starzyk, I. Stan, S. Abelló, A. Bonilla, K. Thomas, C. Fernandez, J.-P. Gilson, J. Pérez-Ramírez, J. Catal. 2009, 264, 11-14.

[18] a) D. Liu, X. Zhang, A. Bhan, M. Tsapatsis, Microporous Mesoporous Mater. 2014, 200, 287-290; b) P. Sazama, B. Wichterlová, Š. Sklenák, V. I. Parvulescu, N. Candu, G. Sádovská, J. Dědeček, P. Klein, V. Pashkova, P. Šťastný, J. Catal. 2014, 318, $22-33$.

[19] D. Liu, A. Bhan, M. Tsapatsis, S. Al Hashimi, ACS Catal. 2011, 1, 7-17.

[20] a) D. Liu, X. Zhang, A. Bhan, M. Tsapatsis, Microporous Mesoporous Mater. 2014, 200, 287-290; b) K. Góra-Marek, K. Tarach, M. Choi, J. Phys.
Chem. C 2014, 118, 12266-12274; c) H. Wei, K. Liu, S. Xie, W. Xin, X. Li, S. Liu, L. Xu, J. Catal. 2013, 307, 103-110.

[21] Z. Wang, P. Dornath, C.-C. Chang, H. Chen, W. Fan, Microporous Mesoporous Mater. 2013, 181, 8-16.

[22] a) H. Mochizuki, T. Yokoi, H. Imai, R. Watanabe, S. Namba, J. N. Kondo, T. Tatsumi, Microporous Mesoporous Mater. 2011, 145, 165-171; b) M. E. Leonowicz, J. A. Lawton, S. L. Lawton, M. K. Rubin, Science 1994, 264, $1910-1913$; c) T. Kugita, S. K. Jana, T. Owada, N. Hashimoto, M. Onaka, S. Namba, Appl. Catal. A 2003, 245, 353-362.

[23] a) J. G. Tittensor, R. J. Gorte, D. M. Chapman, J. Catal. 1992, 138, 714 720 ; b) R. J. Gorte, J. Catal. 1982, 75, 164-174.

[24] a) H. Du, D. H. Olson, J. Phys. Chem. B 2002, 106, 395-400; b) S. Inagaki, K. Kamino, E. Kikuchi, M. Matsukata, Appl. Catal. A 2007, 318, 22-27.

[25] a) W. P. Rothwell, W. X. Shen, J. H. Lunsford, J. Am. Chem. Soc. 1984, 106, $2452-2453$; b) J.-H. Kim, A. Ishida, M. Niwa, React. Kinet. Catal. Lett. 1999, 67, 281-287; c) P. Andy, J. Garcia-Martinez, G. Lee, H. Gonzalez, C. W. Jones, M. E. Davis, J. Catal. 2000, 192, 215-223.

Received: March 21, 2016

Revised: April 29, 2016

Published online on June 23, 2016 